\section{Drug-eluting stents not associated with worse long-term outcome than bare-metal stents}

The use of drug-eluting stents (DESs) in percutaneous coronary intervention reduces the occurrence of restenosis and, consequently, the need for repeat revascularization. The initial randomized studies of DESs focused on their efficacy at reducing restenosis, and were, therefore, inadequately powered to assess differences in the occurrence of less frequent events such as death, myocardial infarction and stent thrombosis. Recent studies have indicated that DESs might be associated with a worse long-term prognosis than bare-metal stents (BMSs). In response to this concern, three research groups have carried out pooled analyses to examine the long-term safety of DESs, as compared with BMSs.

In the first study, Spaulding et al. examined the long-term survival of 1,748 patients enrolled in four randomized trials evaluating sirolimus-eluting stents (SESs) versus BMSs. In these trials, 878 patients underwent placement of an SES and 870 received a BMS. Survival at 4 years was not significantly different between the two groups, and the rates of myocardial infarction and stent thrombosis were also similar. Of note, however, was the significantly lower survival of patients with diabetes treated with SESs compared with patients with diabetes treated with BMSs (hazard ratio for death 2.90, 95\% Cl 1.38-6.10; $P=0.008$ ).

In a second study, Mauri et al. examined the risk of stent thrombosis with DESs versus BMSs in two patient cohorts. The first cohort comprised the same four study populations studied by Spaulding et al., while the second cohort consisted of 1,400 patients treated with a paclitaxel-eluting stent and 1,397 patients treated with a corresponding BMS, enrolled in four different randomized trials. To ensure a uniform evaluation of events across all eight trials, the investigators used a standardized hierarchical definition of stent thrombosis set by the Academic Research Consortium. The researchers found no evidence to indicate an overall increased risk of stent thrombosis in patients with DESs during 4 years of followup. The cumulative incidence of definite or probable stent thrombosis was $1.5 \%$ in the SES group compared with $1.7 \%$ in the corresponding BMS group $(P=0.70)$, and $1.8 \%$ in the paclitaxel group compared with $1.4 \%$ in the corresponding BMS group $(P=0.52)$.

In a third pooled analysis, Kastrati et al. examined the long-term outcome of 4,958 patients enrolled in 14 randomized trials designed to compare SESs with BMSs. Follow-up was in the range 12.1-58.9 months. The researchers found no significant difference in the overall risk of death between those patients who had undergone placement of an SES and those who had undergone BMS placement $(P=0.80)$. By contrast, a significant reduction in the combined end point of death, myocardial infarction or reintervention was seen in those with SESs compared with those with BMSs $(P<0.001)$. The risk of stent thrombosis was similar in the two groups. In agreement with the results produced by Spaulding and coauthors, when mortality was analyzed in the subgroup of patients with diabetes, Kastrati et al. found a trend towards a higher risk of death in those patients with SESs than in those with BMSs.

In conclusion, none of the pooled analyses provides definitive evidence of a worse longterm prognosis with DESs than with BMSs; however, the trend towards an increased risk of death in patients with diabetes who receive an SES justifies further study.

Original articles Spaulding C et al. (2007) A pooled analysis of data comparing sirolimus-eluting stents with bare-metal stents. N Engl J Med 356: 989-997

Mauri L et al. (2007) Stent thrombosis in randomized clinical trials of drug-eluting stents. N Engl J Med 356: 1020-1029

Kastrati A et al. (2007) Analysis of 14 trials comparing sirolimus-eluting stents with bare-metal stents. N Engl J Med 356: $1030-1039$

\section{Large long-term studies of sirolimus-eluting and paclitaxel- eluting stents are inconclusive}

Two recent papers have investigated the safety of drug-eluting stents (DESs), and have also compared the two FDA-approved DESs - sirolimuseluting stents (SESs) and paclitaxel-eluting stents (PESs).

In the first paper, Stone and coauthors report the results of a pooled analysis of doubleblind trials that compared DESs with baremetal stents (BMSs) in the setting of relatively simple lesions. They identified four trials in which patients were randomized to SESs $(n=878)$ or BMSs $(n=870)$, and five trials in which patients were randomized to PESs 